\begin{tabular}{|c|c|}
\hline Title & Simple and efficient finite element analysis of microwave and optical waveguides \\
\hline Author(s) & Koshiba, M.; Inoue, K. \\
\hline Citation & $\begin{array}{l}\text { IEEE Transactions on Microwave Theory and Techniques, 40(2), 371-377 } \\
\text { https://doi.org/10.1109/22.120111 }\end{array}$ \\
\hline Issue Date & $1992-02$ \\
\hline Doc URL & http:/hdl .handle.net/2115/6069 \\
\hline Rights & $\begin{array}{l}\text { () } 1992 \text { IEEE. Personal use of this material is permitted. However, permission to reprint/republish this material for } \\
\text { advertising or promotional purposes or for creating new collective works for resale or redistribution to servers or lists, } \\
\text { or to reuse any copyrighted component of this work in other works must be obtained from the IEEE." } \\
\text { IEEE, IEEE TRA NSA CTIONS ON MICROWA VE THEORY AND TECHNIQUES, volume 31, issue 1, 1992, } \\
\text { pages371-377 }\end{array}$ \\
\hline Tyре & article \\
\hline File Information & ITMTT 40-2.pdf \\
\hline
\end{tabular}

Instructions for use 


\title{
Simple and Efficient Finite-Element Analysis of Microwave and Optical Waveguides
}

\author{
Masanori Koshiba, Senior Member, IEEE, and Kazuhiro Inoue
}

\begin{abstract}
A simple and efficient finite-element method for the analysis of microwave and optical waveguiding problems is formulated using three components of the electric or magnetic field. In order to eliminate spurious solutions, edge elements are introduced. In the edge element approach the nodal parameters are not limited to the magnetic field as in the conventional three-component formulation for the dielectric waveguiding problem. An eigenvalue equation derived here involves only the edge variables in the transversal plane and can provide a direct solution for the propagation constant. To show the vailidity and usefulness of this approach, computed results are illustrated for microstrip transmission lines and dielectric waveguides.
\end{abstract}

\section{INTRODUCTION}

$T^{0}$ RIGOROUSLY evaluate propagation characteristics of microwave and optical waveguides with arbitrarily shaped cross sections, vectorial wave analysis is necessary, and different types of the vector finite-element method (FEM) have been developed. Of the various formulations, the FEM using full vector $\boldsymbol{H}$ field is quite suitable for a wide range of practical, complicated problems [1]-[10]. This approach has been widely used for various dielectric waveguiding structures in microwave, millimeter-wave, and optical wavelength regions, and recently has been utilized as the waveguide solver of CAD packages [7]. The most serious problem associated with this approach is the appearance of spurious solutions. The penalty function method [3], [4], [6], [7] has been used to cure this problem, but in this technique an arbitrary positive constant, called the penalty coefficient, is involved and the accuracy of solutions depends on its magnitude. Furthermore, in the full vectorial formulation [1][10] the propagation constant is first given as an input datum, and subsequently the operating frequency is obtained as a solution. More recently, several methods for solving directly the propagation constant have been developed, but each has its drawback, e.g., a large number of field components [11]-[13], consideration of the adjoint field which does not correspond to the actual electromagnetic field [14], or the need to estimate the line integral in the variational expression [15].

Manuscript received May 14, 1991; revised September 10, 1991. This work was partially supported by a Scientific Research Grant-in-Aid from the Ministry of Education, Science and Culture, Japan.

The authors are with the Department of Electronic Engineering, Hokkaido University, Sapporo, 060 Japan.

IEEE Log Number 9104779.
In this paper a simple and efficient FEM for the analysis of microwave and optical waveguiding problems is formulated using three components of the electric or magnetic field. In order to eliminate spurious solutions and to treat arbitrarily shaped waveguides, triangular edge elements are introduced. An eigenvalue equation derived here involves only the edge variables in the transversal plane and can provide a direct solution for the propagation constant. To show the validity and usefulness of this approach, examples are computed for microstrip transmission lines on isotropic or anisotropic substrates, dielectric rectangular waveguides, and equilateral triangular core waveguides.

\section{Basic Equations}

We consider a dielectric waveguide with a diagonal permittivity tensor and assume that the electromagnetic field in the waveguide varies as $\exp [j(\omega t-\beta z)]$, where $t$ is the time, $z$ is the propagation direction, $\omega$ is the angular frequency, and $\beta$ is the propagation constant in the $z$ direction.

From Maxwell's equations the following vectorial wave equation is derived:

$$
\nabla \times([p] \nabla \times \phi)-k_{0}^{2}[q] \phi=0
$$

with

$$
\begin{aligned}
& {[p]=\left[\begin{array}{ccc}
p_{x} & 0 & 0 \\
0 & p_{y} & 0 \\
0 & 0 & p_{z}
\end{array}\right]} \\
& {[q]=\left[\begin{array}{lll}
q_{x} & 0 & 0 \\
0 & q_{y} & 0 \\
0 & 0 & q_{z}
\end{array}\right]}
\end{aligned}
$$

where $k_{0}$ is the free-space wavenumber, $\phi$ denotes either $\boldsymbol{E}$ or $\boldsymbol{H}$, and the components of $[p]$ and $[q]$ are given by

$$
\begin{aligned}
& p_{x}=p_{y}=p_{z}=1, \\
& q_{x}=\epsilon_{r x}=n_{x}^{2}, \\
& q_{y}=\epsilon_{r y}=n_{y}^{2}, \\
& q_{z}=\epsilon_{r z}=n_{z}^{2} \quad \text { for } \phi=E
\end{aligned}
$$




$$
\begin{aligned}
& p_{x}=1 / \epsilon_{r x}=1 / n_{x}^{2}, \\
& p_{y}=1 / \epsilon_{r y}=1 / n_{y}^{2}, \\
& p_{z}=1 / \epsilon_{r z}=1 / n_{z}^{2}, \\
& q_{x}=q_{y}=q_{z}=1 \quad \text { for } \phi=H .
\end{aligned}
$$

Here $\epsilon_{r x}, \epsilon_{r y}, \epsilon_{r z}$ are the relative permittivities in the $x, y$, $z$ directions, respectively, and $n_{x}, n_{y}, n_{z}$ are the refractive indices in the $x, y, z$ directions, respectively.

The functional for (1) is given by

$$
\begin{aligned}
F= & \iint_{\Omega}\left[(\nabla \times \phi)^{*}\right. \\
& \left.\cdot([p] \nabla \times \phi)-k_{0}^{2}[q] \phi^{*} \cdot \phi\right] d x d y
\end{aligned}
$$

where $\Omega$ is the waveguide cross section and the asterisk denotes complex conjugate.

\section{Finite Element Formulation}

The electromagnetic fields have to be tangentially continuous across material interfaces. In the edge element [5], $[8]-[10],[16]-[20]$, the tangential continuity can be straightforwardly imposed. Hano [5] has developed the FEM with rectangular edge elements for solving inhomogeneous waveguiding problems. Kikuchi [16], on the other hand, has utilized triangular edge elements to treat arbitrarily shaped waveguides, but in [16] only the homogeneous hollow waveguides are analyzed.

In this section we apply the triangular edge element [16], which is different from that used by Hano [8], to inhomogeneous waveguiding problems.

\section{A. Triangular Edge Element}

The six nodes described in the triangular edge element consist of the three corner and three side points as shown in Fig. 1. The corner points 1 to 3 are for the axial component $\phi_{z}\left(E_{z}\right.$ or $\left.H_{z}\right)$, while the side points 4 to 6 are for the tangential component $\phi_{t}\left(E_{t}\right.$ or $\left.H_{t}\right)$.

The axial component $\phi_{z}$ is approximated by a complete polynomial of first order:

$$
\phi_{z}=j\{N(x, y)\}^{T}\left\{\phi_{z}\right\}_{e}=j\{N\}^{T}\left\{\phi_{z}\right\}_{e}
$$

with

$$
\{N\}=\left[\begin{array}{l}
L_{1} \\
L_{2} \\
L_{3}
\end{array}\right]=\frac{1}{2 A_{e}}\left[\begin{array}{lll}
a_{1} & b_{1} & c_{1} \\
a_{2} & b_{2} & c_{2} \\
a_{3} & b_{3} & c_{3}
\end{array}\right]\left[\begin{array}{l}
1 \\
x \\
y
\end{array}\right]
$$

where $\left\{\phi_{z}\right\}_{e}$ is the nodal axial-field vector for each element, $\{N\}$ is the ordinary shape function vector for the linear triangular element, $L_{k}$ 's $(k=1,2,3)$ are the area coordinates, and the area of the element, $A_{e}$, and the coefficients $a_{k}, b_{k}, c_{k}$ are given by

$$
2 A_{e}=\left|\begin{array}{ccc}
1 & 1 & 1 \\
x_{1} & x_{2} & x_{3} \\
y_{1} & y_{2} & y_{3}
\end{array}\right|
$$

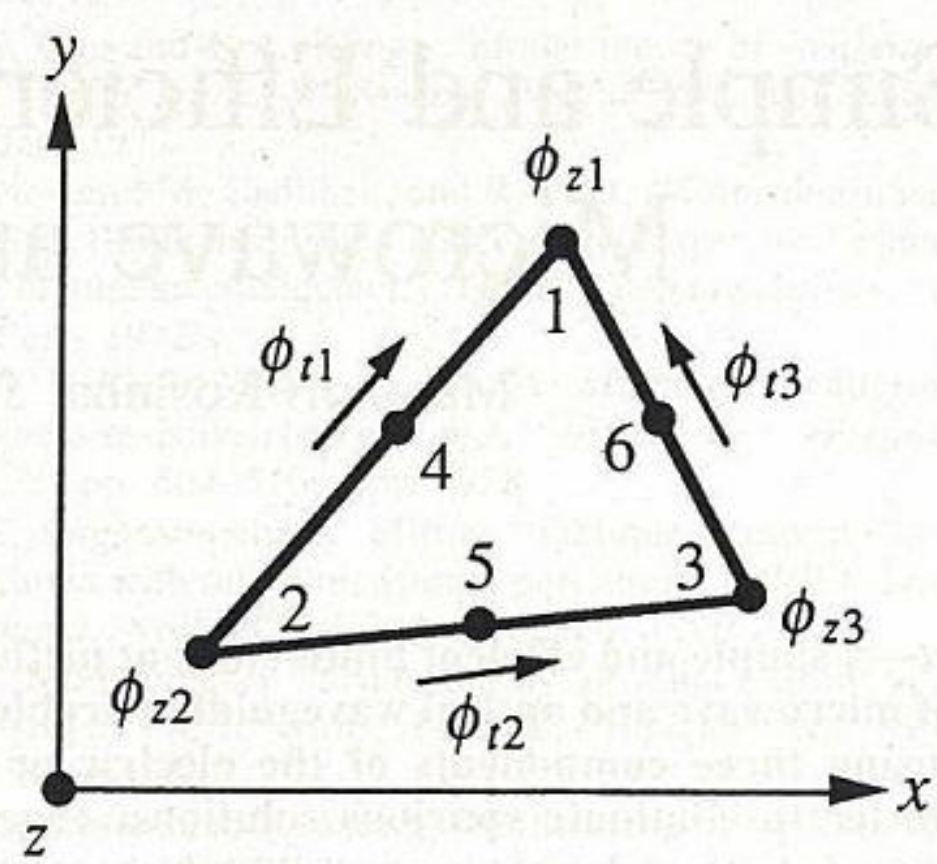

Fig. 1. Triangular edge element.

$$
\begin{aligned}
& a_{k}=x_{l} y_{m}-x_{m} y_{l} \\
& b_{k}=y_{l}-y_{m} \\
& c_{k}=x_{m}-x_{l} .
\end{aligned}
$$

Here $x_{k}, y_{k}(k=1,2,3)$ are the Cartesian coordinates of the corner points 1 to 3 of the triangle and the subscripts $k, l, m$ always progress modulo 3 , i.e., cyclically around the three vertices of the triangle.

The transverse components $\phi_{x}\left(E_{x}\right.$ or $\left.H_{x}\right)$ and $\phi_{y}\left(E_{y}\right.$ or $H_{y}$ ) are approximated by a linear function of $y$ and $x$, respectively:

$$
\begin{aligned}
& \phi_{x}=\{U(y)\}^{T}\left\{\phi_{t}\right\}_{e}=\{U\}^{T}\left\{\phi_{t}\right\}_{e} \\
& \phi_{y}=\{V(x)\}^{T}\left\{\phi_{t}\right\}_{e}=\{V\}^{T}\left\{\phi_{t}\right\}_{e}
\end{aligned}
$$

with

$$
\begin{aligned}
& \{U\}=\left[\begin{array}{l}
\tilde{a}_{1}+\tilde{c}_{1} y \\
\tilde{a}_{2}+\tilde{c}_{2} y \\
\tilde{a}_{3}+\tilde{c}_{3} y
\end{array}\right] \\
& \{V\}=\left[\begin{array}{l}
\tilde{b}_{1}-\tilde{c}_{1} x \\
\tilde{b}_{2}-\tilde{c}_{2} x \\
\tilde{b}_{3}-\tilde{c}_{3} x
\end{array}\right]
\end{aligned}
$$

where $\left\{\phi_{t}\right\}_{e}$ is the edge variables in the transversal plane for each element. $\{U\}$ and $\{V\}$ are the shape function vectors for the triangular edge element, and the coefficients $\tilde{a}_{k}, \tilde{b}_{k}, \tilde{c}_{k}$ are given by

$$
\begin{aligned}
\tilde{a}_{k}= & {\left[\left(y_{m+3} \cos \theta_{m+3}-x_{m+3} \sin \theta_{m+3}\right) \sin \theta_{l+3}\right.} \\
& \left.-\left(y_{l+3} \cos \theta_{l+3}-x_{l+3} \sin \theta_{l+3}\right) \sin \theta_{m+3}\right] / \Delta
\end{aligned}
$$

$$
\begin{aligned}
\tilde{b}_{k}= & {\left[\left(y_{l+3} \cos \theta_{l+3}-x_{l+3} \sin \theta_{l+3}\right) \cos \theta_{m+3}\right.} \\
& \left.-\left(y_{m+3} \cos \theta_{m+3}-x_{m+3} \sin \theta_{m+3}\right) \cos \theta_{l+3}\right] / \Delta
\end{aligned}
$$

$$
\tilde{c}_{k}=\left(\cos \theta_{l+3} \sin \theta_{m+3}-\cos \theta_{m+3} \sin \theta_{l+3}\right) / \Delta
$$


with

$$
\begin{aligned}
0 \leq & \theta_{k+3}=\tan ^{-1}\left\{\left(y_{k}-y_{l}\right) /\left(x_{k}-x_{l}\right)\right\}<\pi \\
\Delta= & \sum_{k=1}^{3}\left(y_{k+3} \cos \theta_{k+3}-x_{k+3} \sin \theta_{k+3}\right) \\
& \cdot\left(\cos \theta_{l+3} \sin \theta_{m+3}-\cos \theta_{m+3} \sin \theta_{l+3}\right) .
\end{aligned}
$$

Here $x_{k+3}, y_{k+3}(k=1,2,3)$ are the Cartesian coordinates of the side points 4 to 6 of the triangle.

Note that the tangential component, $\phi_{t}=\phi_{x} \cos \theta+$ $\phi_{y} \sin \theta$, is continuous along the interelement boundaries and is constant on each side of triangles.

\section{B. Finite-Element Discretization}

Dividing the waveguide cross section into a number of edge elements, we expand the transverse components $\phi_{x}$, $\phi_{y}$ and the axial component $\phi_{z}$ in each element as

$$
\phi=[N]^{T}\{\phi\}_{e}
$$

with

$$
\begin{aligned}
\{\phi\}_{e} & =\left[\begin{array}{l}
\left\{\phi_{t}\right\}_{e} \\
\left\{\phi_{z}\right\}_{e}
\end{array}\right] \\
{[N] } & =\left[\begin{array}{lll}
\{U\} & \{V\} & \{0\} \\
\{0\} & \{0\} & j\{N\}
\end{array}\right]
\end{aligned}
$$

where $\{0\}$ is a null vector.

Substituting (22) into (6), from the variational principle we obtain the following eigenvalue problem:

$$
[K]\{\phi\}-k_{0}^{2}[M]\{\phi\}=\{0\}
$$

with

$$
\begin{aligned}
& {[K]=\left[\begin{array}{ll}
{\left[K_{t t}\right]} & {\left[K_{t z}\right]} \\
{\left[K_{z t}\right]} & {\left[K_{z z}\right]}
\end{array}\right]} \\
& =\sum_{e} \iint_{e}[B] *[p][B]^{T} d x d y \\
& {[M]=\left[\begin{array}{ll}
{\left[M_{t t}\right]} & {[0]} \\
{[0]} & {\left[M_{z z}\right]}
\end{array}\right]} \\
& =\sum_{e} \iint_{e}[N] *[q][N]^{T} d x d y \\
& {[B]=\left[\begin{array}{ccc}
j \beta\{V\} & -j \beta\{U\} & -\left\{U_{y}\right\}+\left\{V_{x}\right\} \\
j\left\{N_{y}\right\} & -j\left\{N_{x}\right\} & \{0\}
\end{array}\right]}
\end{aligned}
$$

where $\{\phi\}$ is the global field vector and the submatrices of $[K]$ and $[M]$ are given by

$$
\left[K_{t t}\right]=\sum_{e} \int_{e} \int_{e}\left[p_{x} \beta^{2}\{V\}\{V\}^{T}+p_{y} \beta^{2}\{U\}\{U\}^{T}\right.
$$$$
\left.+4 p_{z}\left\{U_{y}\right\}\left\{U_{y}\right\}^{T}\right] d x d y
$$

$$
\begin{aligned}
{\left[K_{t z}\right]=} & {\left[K_{z t}\right]^{T} } \\
= & \sum_{e} \iint_{e}\left[p_{x} \beta\{V\}\left\{N_{y}\right\}^{T}\right. \\
& \left.+p_{y} \beta\{U\}\left\{N_{x}\right\}^{T}\right] d x d y
\end{aligned}
$$

$\left[K_{z z}\right]=\sum_{e} \iint_{e}\left[p_{x}\left\{N_{y}\right\}\left\{N_{y}\right\}^{T}+p_{y}\left\{N_{x}\right\}\left\{N_{x}\right\}^{T}\right] d x d y$

$\left[M_{t t}\right]=\sum_{e} \iint_{e}\left[q_{x}\{U\}\{U\}^{T}+q_{y}\{V\}\{V\}^{T}\right] d x d y$

$\left[M_{z z}\right]=\sum_{e} \iint_{e} q_{z}\{N\}\{N\}^{T} d x d y$.

Here $\left\{N_{x}\right\} \equiv \partial\{N\} / \partial x,\left\{N_{y}\right\} \equiv \partial\{N\} / \partial y,\left\{U_{y}\right\} \equiv$ $d\{U\} / d y$, and $\left\{V_{x}\right\} \equiv d\{V\} / d x$.

Equation (25) may be rewritten as

$$
\begin{array}{r}
{\left[K_{t t}\right]\left\{\phi_{t}\right\}-\beta\left[K_{t z}\right]\left\{\phi_{z}\right\}-\beta^{2}\left[M_{t t}\right]\left\{\phi_{t}\right\}=\{0\}} \\
-\beta\left[K_{z t}\right]\left\{\phi_{t}\right\}+\left[K_{z z}\right]\left\{\phi_{z}\right\}=\{0\}
\end{array}
$$

with

$$
\begin{aligned}
{\left[K_{t t}\right]=} & \sum_{e} \int_{e} \int_{e}\left[q_{x} k_{0}^{2}\{U\}\{U\}^{T}+q_{y} k_{0}^{2}\{V\}\{V\}^{T}\right. \\
& \left.-4 p_{z}\left\{U_{y}\right\}\left\{U_{y}\right\}^{T}\right] d x d y \\
{\left[K_{t z}\right]=} & {\left[K_{z t}\right]^{T} } \\
= & \sum_{e} \int_{e}\left[p_{x}\{V\}\left\{N_{y}\right\}^{T}+p_{y}\{U\}\left\{N_{x}\right\}^{T}\right] d x d y \\
{\left[K_{z z}\right]=} & \sum_{e} \int_{e}\left[q_{z} k_{0}^{2}\{N\}\{N\}^{T}-p_{x}\left\{N_{y}\right\}\left\{N_{y}\right\}^{T}\right. \\
& \left.-p_{y}\left\{N_{x}\right\}\left\{N_{x}\right\}^{T}\right] d x d y \\
{\left[M_{t t}\right]=} & \sum_{e} \iint_{e}\left[p_{x}\{V\}\{V\}^{T}+p_{y}\{U\}\{U\}^{T}\right] d x d y .
\end{aligned}
$$

Note that the submatrices in (32) and (33) are different from those in (29) and (30).

Substituting (31b) into (31a), we obtain the following final eigenvalue problem:

$$
\left[K_{t t}\right]\left\{\phi_{t}\right\}-\beta^{2}\left[\tilde{M}_{t t}\right]\left\{\phi_{t}\right\}=\{0\}
$$

with

$$
\left[\tilde{M}_{t t}\right]=\left[M_{t t}\right]+\left[K_{t z}\right]\left[K_{z z}\right]^{-1}\left[K_{z t}\right]
$$


Note that (34) will give a solution directly for the propagation constant and the corresponding field distribution, and involves only the edge variables in the transversal plane $\left\{\phi_{t}\right\}$. But it is important to point out the price paid for this: a matrix inversion has to be performed and the sparsity of the matrices is destroyed.

The integrals necessary to construct element matrices are summarized in the Appendix.

\section{NUMERICAL EXAMPLES}

First, we consider a microstrip transmission line in Fig. 2 and subdivide one-half of the waveguide cross section into edge elements, where $W=1.27 \mathrm{~mm}, t=0, h=$ $1.27 \mathrm{~mm}, X=12.7 \mathrm{~mm}$, and $Y=12.7 \mathrm{~mm}$. Fig. 3(a) and (b) show the propagation characteristics for the first two modes of a microstrip on an isotropic substrate with $\epsilon_{r}=8.875$ and for those of a microstrip on an anisotropic substrate with $\epsilon_{r x}=\epsilon_{r z}=9.4$ and $\epsilon_{r y}=11.6$, respectively, where the number of elements $N_{E}=364$, the number of corner points $N_{C}=210$, and the number of side points $N_{S}=573$. Our results agree well with previously reported ones for both isotropic [21]-[23] and anisotropic [23], [24] cases.

Next, we consider a dielectric rectangular waveguide in Fig. 4 , where $n_{1}$ and $n_{2}$ are the refractive indices of the core and cladding regions, respectively. Because of the twofold symmetry of the system, we subdivide only onequarter of the waveguide cross section into edge elements. For simplicity, assuming the artificial boundaries $x= \pm X / 2$ and $y= \pm Y / 2$ far from the core region, the original unbounded structure is replaced by a corresponding bounded one. Here, the conditions for the perfect electric or perfect magnetic conductors are imposed suitably on the artificial boundaries, so as not to restrict the dominant electromagnetic field component there. Fig. 5 shows the propagation characteristics of this waveguide, where $W=2 t, X=10 t, Y=5 t, N_{E}=320, N_{C}=187$, $N_{S}=506$, and the normalized frequency $v$ and the normalized propagation constant $b$ are defined as

$$
\begin{gathered}
v=k_{0} t \sqrt{n_{1}^{2}-n_{2}^{2}} / \pi \\
b=\frac{\left(\beta / k_{0}\right)^{2}-n_{2}^{2}}{n_{1}^{2}-n_{2}^{2}} .
\end{gathered}
$$

Our results agree well with the results of the point matching method [25]. The results of the Marcatili's method [26] deviate from those of the point matching method at lower frequencies.

Lastly, we consider an equilateral triangular core waveguide in Fig. 6 and subdivide one-half of the waveguide cross section into edge elements. Fig. 7 shows the propagation characteristics for the $E_{11}^{y}$ mode of this waveguide, where $X=6 t, Y=5 t, N_{E}=360, N_{C}=208$, and $N_{S}=567$. The finite-element solutions of edge element formulation agree well with those of axial-field ( $E_{z}$ and $\mathrm{H}_{z}$ ) formulation [27] and those of full vector $H$-field formulation with the penalty coefficient $s=1[28]$.

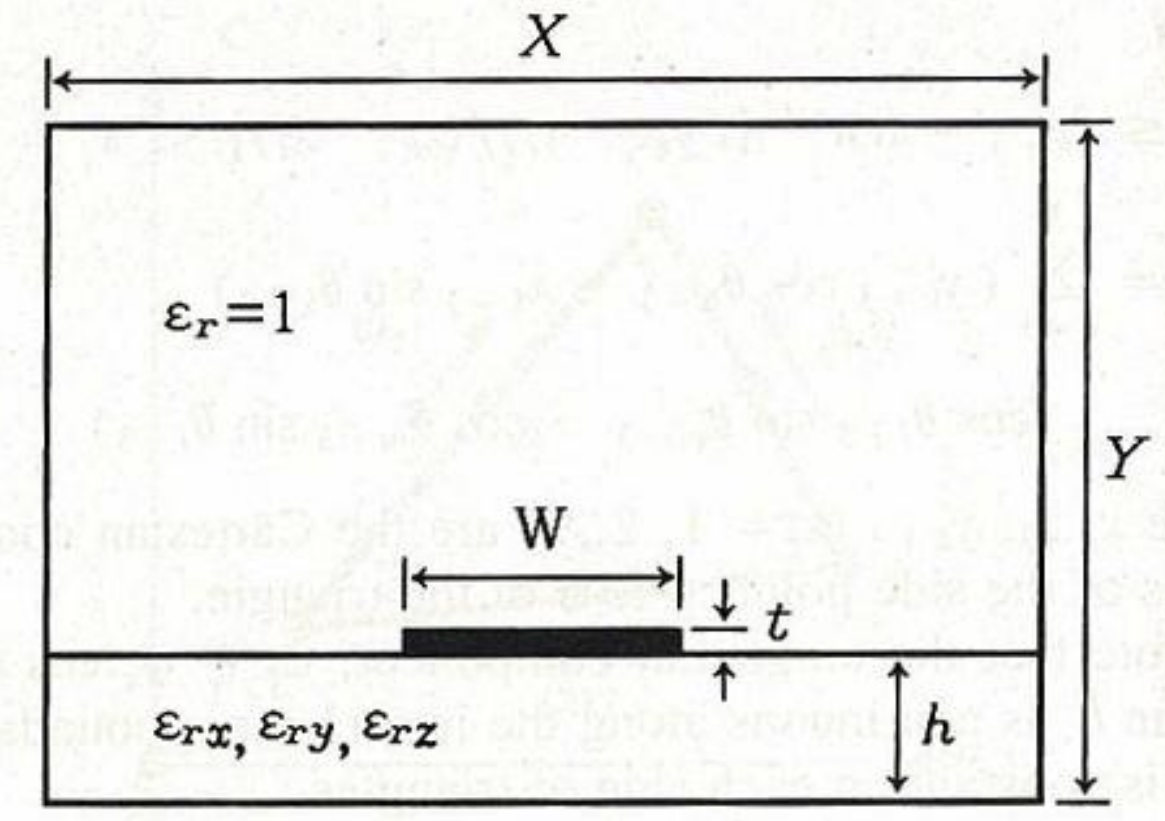

Fig. 2. Shielded microstrip transmission line.

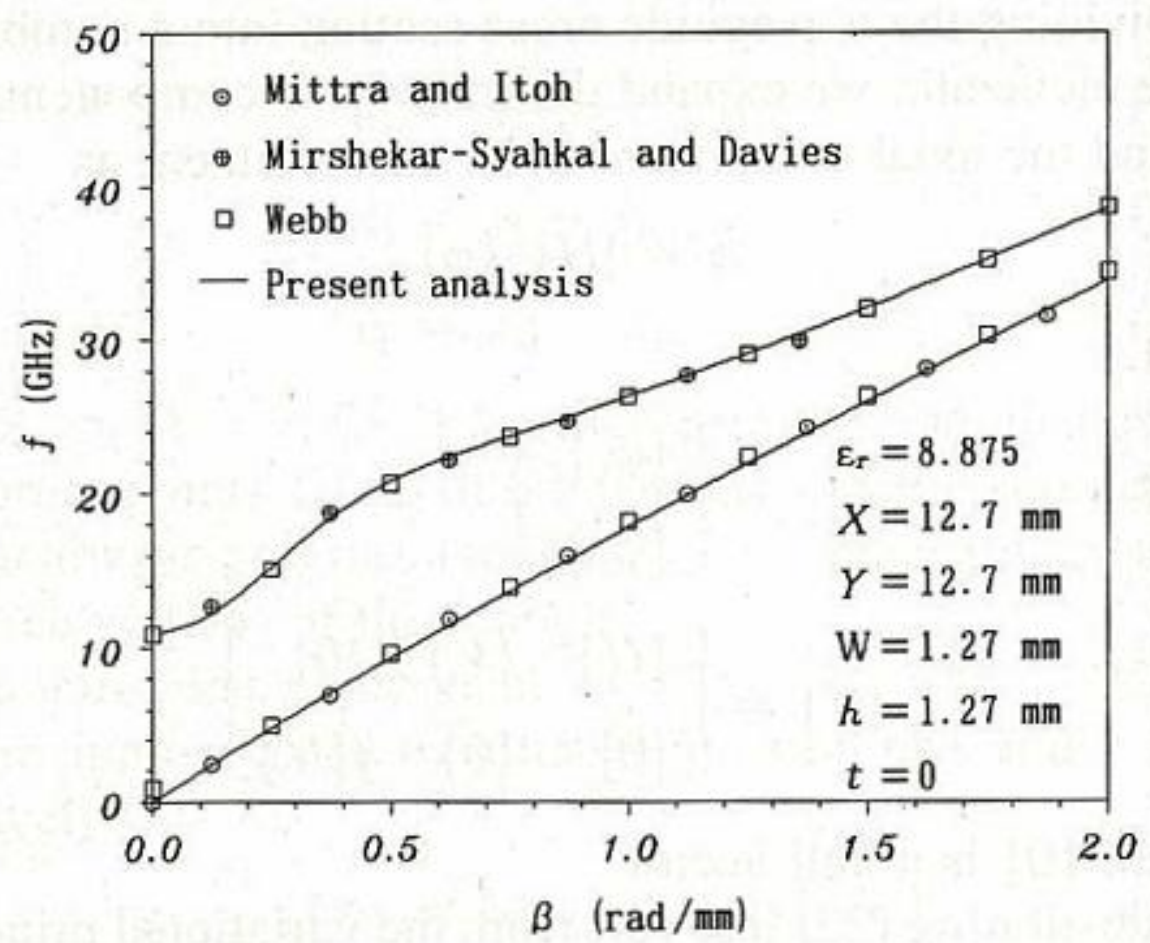

(a)

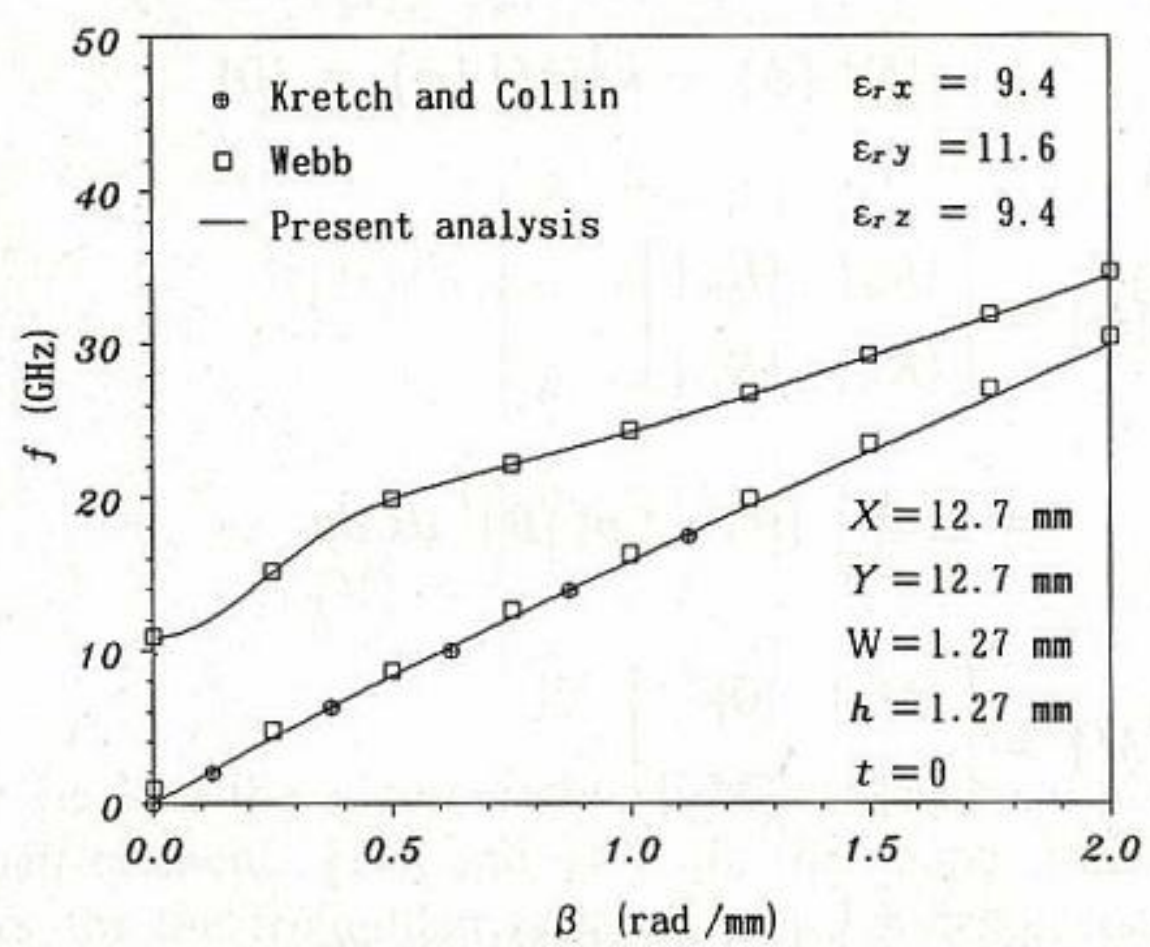

(b)

Fig. 3. Propagation characteristics of a microstrip transmission line. (a) Isotropic substrate. (b) Anisotropic substrate.

Note that the spurious solutions are included in the finite-element solutions of axial-field formulation. To avoid confusion, such spurious solutions are not shown in Fig. 7(a). In the edge element method spurious solutions do not appear anywhere. Furthermore, the newly derived eigenvalue problem (34) does not produce zero eigenvalues [5], [8]-[10] which are present in (25). The convergence of solutions has been checked by increasing the number 


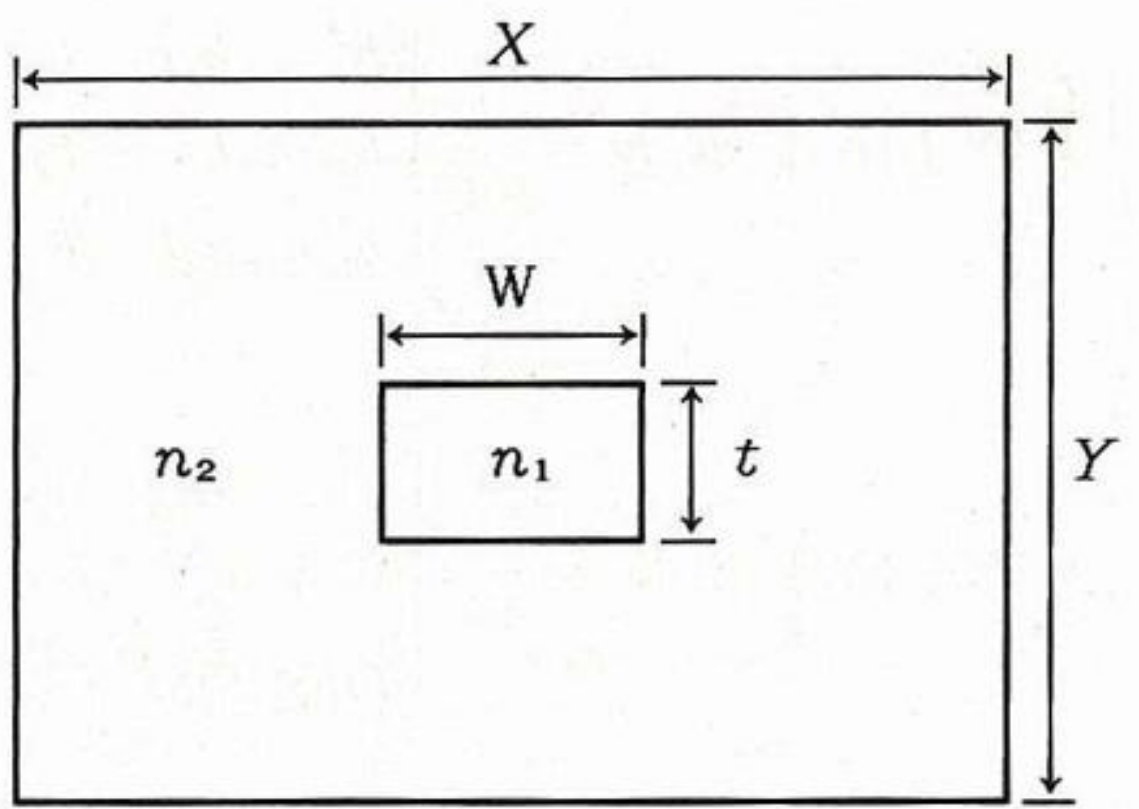

Fig. 4. Dielectric rectangular waveguide.

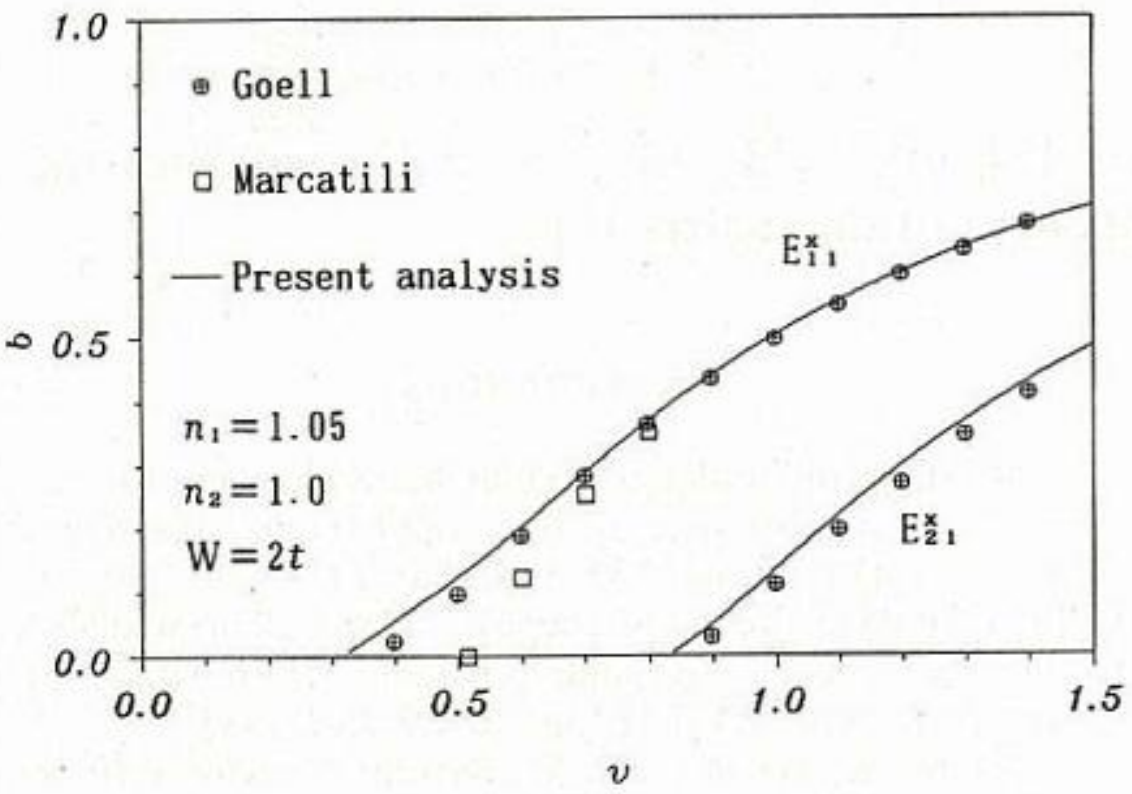

(a)

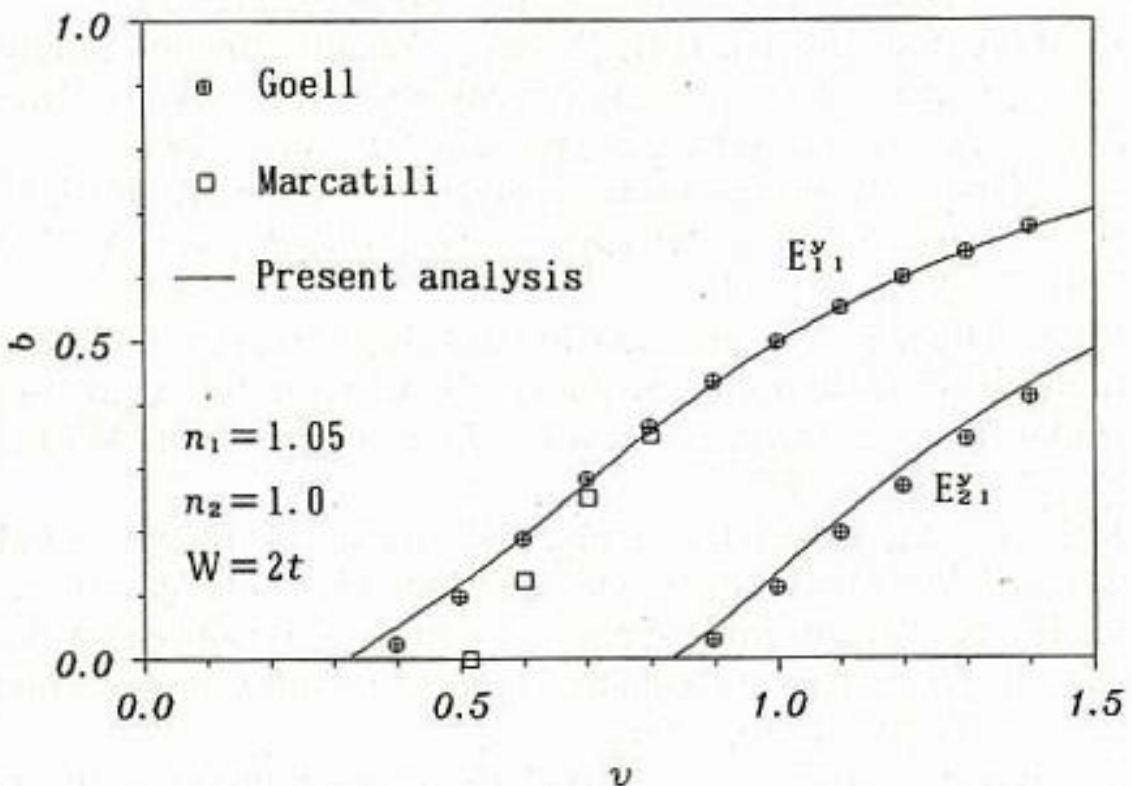

(b)

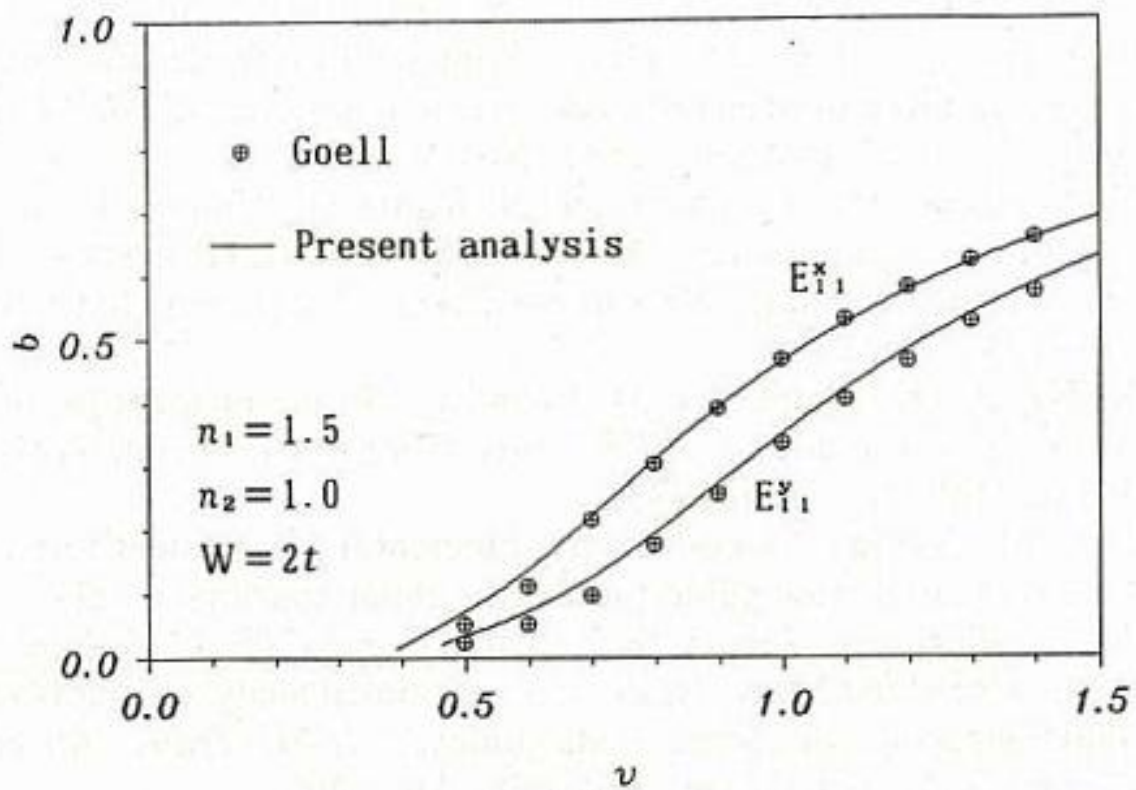

(c)

Fig. 5. Propagation characteristics of a dielectric rectangular waveguide. (a) $E_{11}^{x}$ and $E_{21}^{x}$ modes $\left(n_{1}=1.05, n_{2}=1.0\right)$. (b) $E_{11}^{y}$ and $E_{21}^{y}$ modes $\left(n_{1}\right.$ $\left.=1.05, n_{2}=1.0\right)$. (c) $E_{11}^{x}$ and $E_{11}^{y}$ modes $\left(n_{1}=1.5, n_{2}=1.0\right)$.

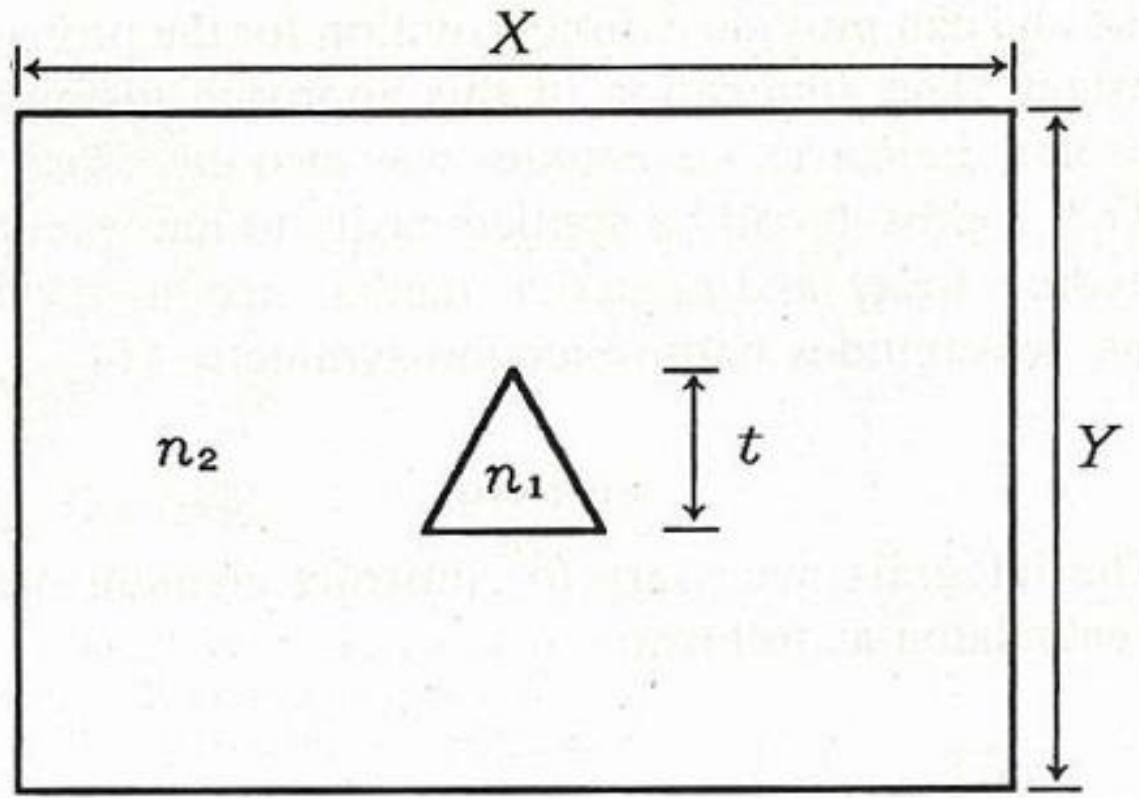

Fig. 6. Equilateral triangular core waveguide.

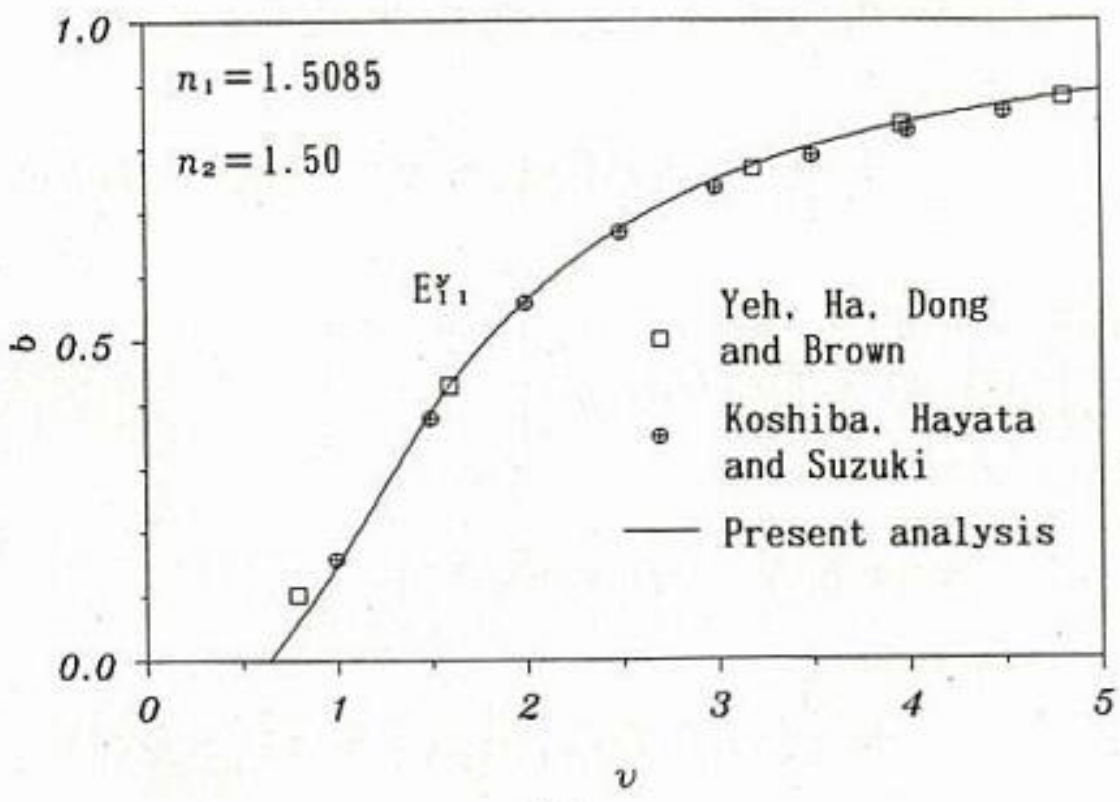

(a)

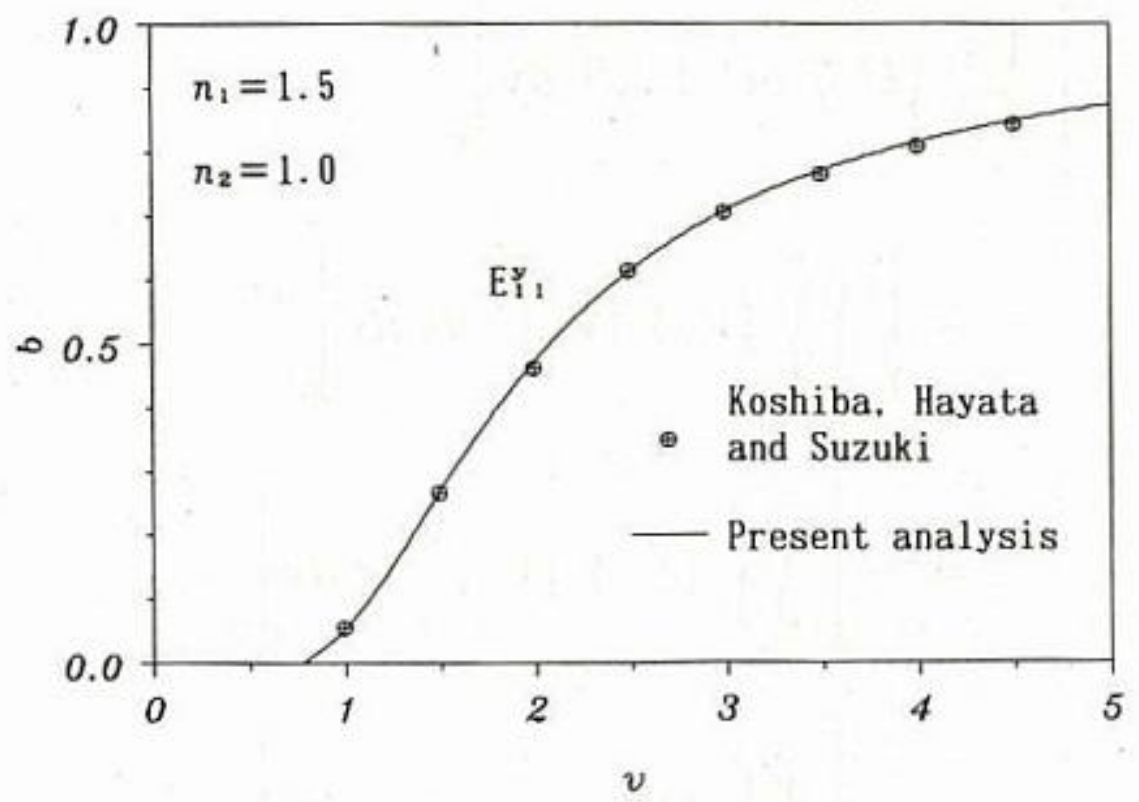

(b)

Fig. 7. Propagation characteristics for the $E_{11}^{y}$ mode of an equilateral triangular core waveguide. (a) $n_{1}=1.5085$ and $n_{2}=1.50$. (b) $n_{1}=1.5$ and $n_{2}=1.0$.

of elements and the values of $X$ and $Y$ for the influence of the artificial boundaries to be negligible.

\section{CONCLUSION}

A simple and efficient finite-element method for the analysis of microwave and optical waveguiding problems was formulated using three components of the electric or magnetic field. In order to eliminate spurious solutions and to treat arbitrarily shaped waveguides, triangular edge elements were utilized. An eigenvalue equation derived here involves only the edge variables in the transversal 
plane and can provide a direct solution for the propagation constant. The application of this approach to microstrip lines and dielectric waveguides was also discussed.

This approach can be applied easily to the waveguides including lossy and/or active media, and to the anisotropic waveguides with reflection symmetry [14], [15].

\section{APPENDIX}

The integrals necessary to construct element matrices are calculated as follows:

$$
\begin{aligned}
& {\left[\iint_{e}\{U\}\{U\}^{T} d x d y\right]_{k l}} \\
& =A_{e} \tilde{a}_{k} \tilde{a}_{l}+A_{e} y_{c}\left(\tilde{a}_{k} \tilde{c}_{l}+\tilde{c}_{k} \tilde{a}_{l}\right) \\
& \quad+\frac{1}{12} A_{e} \tilde{c}_{k} \tilde{c}_{l}\left(y_{1}^{2}+y_{2}^{2}+y_{3}^{2}+9 y_{c}^{2}\right)
\end{aligned}
$$

$$
\begin{aligned}
& {\left[\iint_{e}\{V\}\{V\}^{T} d x d y\right]_{k l}} \\
& =A_{e} \tilde{b}_{k} \tilde{b}_{l}-A_{e} x_{c}\left(\tilde{b}_{k} \tilde{c}_{l}+\tilde{c}_{k} \tilde{b}_{l}\right) \\
& \quad+\frac{1}{12} A_{e} \tilde{c}_{k} \tilde{c}_{l}\left(x_{1}^{2}+x_{2}^{2}+x_{3}^{2}+9 x_{c}^{2}\right)
\end{aligned}
$$$$
\left[\iint_{e}\left\{U_{y}\right\}\left\{U_{y}\right\}^{T} d x d y\right]_{k l}
$$$$
=\left[\iint_{e}\left\{V_{x}\right\}\left\{V_{x}\right\}^{T} d x d y\right]_{k l}
$$$$
=-\left[\iint_{e}\left\{U_{y}\right\}\left\{V_{x}\right\}^{T} d x d y\right]_{k l}
$$$$
=-\left[\iint_{e}\left\{V_{x}\right\}\left\{U_{y}\right\}^{T} d x d y\right]_{k l}
$$$$
=A_{e} \tilde{c}_{k} \tilde{c}_{l}
$$

$\left[\int\right.$
$\left[\int\right.$

$\left.\iint_{e}\{U\}\left\{N_{x}\right\}^{T} d x d y\right]_{k l}=\frac{1}{2}\left(\tilde{a}_{k}+\tilde{c}_{k} y_{c}\right) b_{l}$

$\left.\iint_{e}\{V\}\left\{N_{y}\right\}^{T} d x d y\right]_{k l}=\frac{1}{2}\left(\tilde{b}_{k}-\tilde{c}_{k} x_{c}\right) c_{l}$

$\iint_{e}\{N\}\{N\}^{T} d x d y=\frac{A_{e}}{12}\left[\begin{array}{lll}2 & 1 & 1 \\ 1 & 2 & 1 \\ 1 & 1 & 2\end{array}\right]$

$$
\iint_{e}\left\{N_{x}\right\}\left\{N_{x}\right\}^{T} d x d y=\frac{1}{4 A_{e}}\left[\begin{array}{lll}
b_{1}^{2} & b_{1} b_{2} & b_{1} b_{3} \\
b_{1} b_{2} & b_{2}^{2} & b_{2} b_{3} \\
b_{1} b_{3} & b_{2} b_{3} & b_{3}^{2}
\end{array}\right]
$$

$$
\iint_{e}\left\{N_{y}\right\}\left\{N_{y}\right\}^{T} d x d y=\frac{1}{4 A_{e}}\left[\begin{array}{lll}
c_{1}^{2} & c_{1} c_{2} & c_{1} c_{3} \\
c_{1} c_{2} & c_{2}^{2} & c_{2} c_{3} \\
c_{1} c_{3} & c_{2} c_{3} & c_{3}^{2}
\end{array}\right]
$$

with

$$
\begin{aligned}
& x_{c}=\left(x_{1}+x_{2}+x_{3}\right) / 3 \\
& y_{c}=\left(y_{1}+y_{2}+y_{3}\right) / 3
\end{aligned}
$$

where $[\cdot]_{k l}(k l=11,12, \cdots, 33)$ indicates the $(k, l)$ component of the matrix $[\cdot]$.

\section{REFERENCES}

[1] A. Konrad, "High-order triangular finite elements for electromagnetic waves in anisotropic media," IEEE Trans. Microwave Theory Tech., vol. MTT-25, pp. 353-360, May 1977.

[2] B. M. A. Rahman and J. B. Davies, "Finite-element analysis of optical and microwave waveguide problem,"' IEEE Trans. Microwave Theory Tech., vol. MTT-32, pp. 20-28, Jan. 1984.

[3] M. Koshiba, K. Hayata, and M. Suzuki, "Vectorial finite-element formulation without spurious solutions for dielectric waveguide problems," Electron. Lett., vol. 20, pp. 409-410, May 1984.

[4] B. M. A. Rahman and J. B. Davies, "Penalty function improvement of waveguide solution by finite elements," IEEE Trans. Microwave Theory Tech., vol. MTT-32, pp. 922-928, Aug. 1984.

[5] M. Hano, "Finite-element analysis of dielectric-loaded waveguides," IEEE Trans. Microwave Theory Tech., vol. MTT-32, pp. 1275-1279, Oct. 1984.

[6] M. Koshiba, K. Hayata, and M. Suzuki, "Improved finite-element formulation in terms of magnetic field vector for dielectric waveguides," IEEE Trans. Microwave Theory Tech., vol. MTT-33, pp. 227-233, Mar. 1985.

[7] T. P. Young, "Design of integrated optical circuits using finite elements," IEE Proc., pt. A, vol. 135, pp. 135-144, Mar. 1988.

[8] M. Hano, "Vector finite-element solution of anisotropic waveguides using novel triangular elements," Electron. and Commun. Japan, pt. 2 , vol. 71, pp. 71-80, 1988.

[9] R. Miniowitz and J. P. Webb, "Covariant-projection quadrilateral elements for the analysis of waveguides with sharp edges," IEEE Trans. Microwave Theory Tech., vol. 39, pp. 501-505, Mar. 1991.

[10] S. H. Wong and Z. J. Cendes, "Combined finite element modal solution of three-dimensional eddy current problems," IEEE Trans. Magn., vol. 24, pp. 2685-2687, Nov. 1988.

[11] T. Angkaew, M. Matsuhara, and N. Kumagai, "Finite-element analysis of waveguide modes: A novel approach that eliminates spurious modes," IEEE Trans. Microwave Theory Tech., vol. MTT-35, pp. 117-123, Feb. 1987.

[12] K. Hayata, K. Miura, and M. Koshiba, "Finite-element formulation for lossy waveguides," IEEE Trans. Microwave Theory Tech., vol. 36, pp. 268-276, Feb. 1988.

[13] J. A. M. Svedin, "A numerically efficient finite-element formulation for the general waveguide problem without spurious modes," IEEE Trans. Microwave Theory Tech., vol. 37, pp. 1708-1715, Nov. 1989.

[14] W. C. Chew and M. A. Nasir, "A variational analysis of anisotropic, inhomogeneous dielectric waveguides," IEEE Trans. Microwave Theory Tech., vol. 37, pp. 661-668, Apr. 1989.

[15] F. A. Fernandez and Y. Lu, "Variational finite element analysis of dielectric waveguides with no spurious solutions," Electron. Lett., vol. 26, pp. 2125-2126, Dec. 1990.

[16] F. Kikuchi, "Mixed and penalty formulations for finite element anal- 
ysis of an eigenvalue problem in electromagnetism," Comput. Methods Appl. Mech. Eng., vol. 64, pp. 509-521, 1987.

[17] A. Bossavit, "A rationale for "edge-elements" in 3-D fields computations," IEEE Trans. Magn., vol. 24, pp. 74-79, Jan. 1988.

[18] — , "Whitney forms: a class of finite elements for three-dimensional computations in electromagnetism," IEE Proc., pt. A, vol. 135, pp. 493-500, Nov. 1988.

[19] A. Kameari, "Calculation of transient 3D eddy current using edgeelements,"'IEEE Trans. Magn., vol. 26, pp. 466-469, Mar. 1990.

[20] A. Bossavit, "Solving Maxwell equations in a closed cavity, and the question of 'spurious modes',"' IEEE Trans. Magn., vol. 26, pp. 702705, Mar. 1990.

[21] R. Mittra and T. Itoh, "A new technique for the analysis of the dispersion characteristics of microstrip lines," IEEE Trans. Microwave Theory Tech., vol. MTT-19, pp. 47-56, Jan. 1971.

[22] D. Mirshekar-Syahkal and J. B. Davies, "Accurate solution of microstrip and coplanar structures for dispersion and for dielectric and conductor losses," IEEE Trans. Microwave Theory Tech., vol. MTT27, pp. 694-699, July 1979.

[23] J. P. Webb, "Finite element analysis of dispersion in waveguides with sharp metal edges," IEEE Trans. Microwave Theory Tech., vol. 36, pp. 1819-1824, Dec. 1988.

[24] B. E. Kretch and R. E. Collin, "Microstrip dispersion including anisotropic substrates," IEEE Trans. Microwave Theory Tech., vol. MTT-35, pp. 710-718, Aug. 1987.

[25] J. E. Goell, "A circular harmonic computer analysis of rectangular dielectric waveguides," Bell Syst. Tech. J., vol. 48, pp. 2133-2160, Sept. 1969.

[26] E. A. J. Marcatili, "Dielectric rectangular waveguide and directional coupler for integrated optics,' Bell Syst. Tech. J., vol. 48, pp. 2071 2102 , Sept. 1969.

[27] C. Yeh, K. Ha, S. B. Dong, and W. P. Brown, "Single-mode optical waveguides," Appl. Opt., vol. 18, pp. 1490-1504, May 1979.

[28] M. Koshiba, K. Hayata, and M. Suzuki, "Vector-E field finite-element analysis of dielectric optical waveguides," Appl. Opt., vol. 25, pp. 10-11, Jan. 1986.

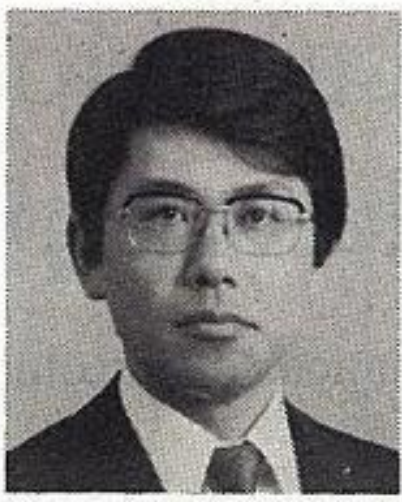

Masanori Koshiba (SM'84) was born in Sapporo, Japan, on November 23, 1948. He received the B.S., M.S., and Ph.D. degrees in electronic engineering from Hokkaido University, Sapporo, Japan, in 1971, 1973, and 1976, respectively.

In 1976, he joined the Department of Electronic Engineering, Kitami Institute of Technology, Kitami, Japan. From 1979 to 1987, he was an Associate Professor of Electronic Engineering at Hokkaido University, and in 1987 he became a Professor there. He has been engaged in research on lightwave technology, surface acoustic waves, magnetostatic waves, microwave field theory, and applications of finite-element and boundaryelement methods to field problems.

Dr. Koshiba is a member of the Institute of Electronics, Information and Communication Engineers (IEICE), the Institute of Television Engineers of Japan, the Institute of Electrical Engineers of Japan, the Japan Society for Simulation Technology, and the Japan Society for Computational Methods in Engineering. In 1987, he was awarded the 1986 Paper Award by the IEICE.

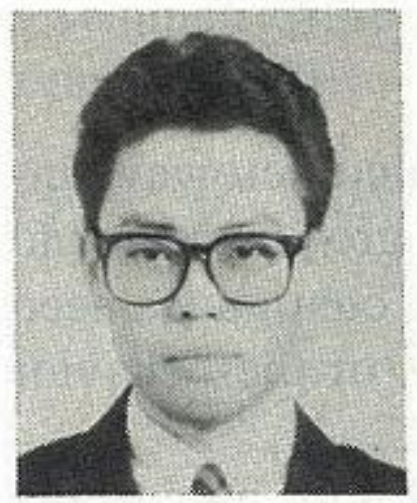

Kazuhiro Inoue was born in Chitose, Hokkaido, Japan, on January 10, 1965. He received the B.S. and M.S. degrees in electronic engineering from Hokkaido University, Sapporo, Japan, in 1987 and 1989 , respectively. He is presently studying toward the $\mathrm{Ph} . \mathrm{D}$. degree in electronic engineering at Hokkaido University.

$\mathrm{Mr}$. Inoue is a member of the Institute of Electronics, Information and Communication Engineers (IEICE). 